\title{
ON THE GLOBAL CONTROLLABILITY STRUCTURE OF NONLINEAR SYSTEMS
}

\author{
F. COLONIUS AND W. KLIEMANN
}

\section{INTRODUCTION}

Complete controllability is a rather rare event for nonlinear systems with restricted control range. In many applications it is therefore interesting to find the subsets of the state space, in which the system is controllable, that is, the control sets, and the relations between them. As shown in [3], generically, all trajectories enter invariant control sets as time tends to infinity. Hence our philosophy in the global description of control systems is that invariant control sets are sinks' (and open control sets are sources). Here we consider the corresponding domains of attraction and their intersections, and characterize them via relatively invariant control sets. More generally, we discuss the existence of control sets in subsets $L$ of the state space, which are not positively invariant under all controls. ${ }^{1}$

\section{Preliminaries}

We will first describe the considered class of control systems:

$$
\begin{gathered}
\dot{x}(t)=X(x(t), u(t)), t \in \mathbb{R} \\
u \in \mathcal{U}_{p c}=\left\{\begin{array}{c}
u: \mathbb{R} \rightarrow \mathbb{R}^{m} \mid u(t) \in U \text { for all } \\
t \in \mathbb{R}, \text { piecewise constant }
\end{array}\right\}
\end{gathered}
$$

where $X: M \times \mathbb{R}^{m} \rightarrow \mathbf{T} M$ is $C^{\infty}$. We assume that $F=\{X(\cdot, u) \mid u \in U\}$ consists of complete vector fields on a connected paracompact manifold $M$ of dimension $d$ and that the control range $U \subset \mathbb{R}^{m}$ is nonvoid. Hence for every initial state $x \in M$ and every control function $u \in \mathcal{U}_{p c}$ there exists a unique solution of (2.1). This solution will be denoted by $\varphi(t, x, u), t \in \mathbb{R}$, satisfying $\varphi(0, x, u)=x$. We define the set of points reachable from $x$ and controllable to $x$ with piecewise constant controls in the following way.

Definition 1. For $x \in M$ the set of points reachable from $x$ up to time $T>0$ is

$$
\mathcal{O}_{\leq T}^{+}(x)=\left\{\begin{array}{c}
y \in M \mid \text { there are } 0 \leq t \leq T \\
\text { and } u \in \mathcal{U}_{p c} \text { with } y=\varphi(t, x, u)
\end{array}\right\}
$$

The set of points controllable to $x$ within time $T>0$ is

$$
\mathcal{O}_{\leq T}^{-}(x)=\left\{\begin{array}{c}
y \in M \mid \text { there are } 0 \leq t \leq T \\
\text { and } u \in \mathcal{U}_{p c} \text { with } x=\varphi(t, y, u)
\end{array}\right\}
$$

Furthermore let $\mathcal{O}^{+}(x)=\bigcup_{T>0} \mathcal{O}_{\leq T}^{+}(x)$ and $\mathcal{O}^{-}(x)=\bigcup_{T>0} \mathcal{O}_{\leq T}^{-}(x)$ denote the reachable set from $x$ and the set controllable to $x$, respectively.

\footnotetext{
${ }^{1}$ Partially supported by ONR grant no. N00014-96-1-0279 and DFG Co 124/12-2
} 
Frequently, we will require local accessibility from $x \in M$, that is $\mathcal{O}_{<T}^{+}(x)$ and $\mathcal{O}_{\leq T}^{-}(x)$ have nonvoid interior for all $T>0$. The system (2.1) is called locally accessible, if every point in its state space has this property. Recall that local accessibility is guaranteed by the accessibility rank condition

$$
\operatorname{dim} \mathcal{L} \mathcal{A}\{X(\cdot, u) \mid u \in U\}(x)=d \text { for all } x \in M
$$

which can in principle be checked for a given system.

Next we formally define the regions of complete controllability.

Definition 2. A set $D \subset M$ with nonvoid interior is called a control set of system (2.1) if for all $x \in D$ one has $D \subset \operatorname{cl} \mathcal{O}^{+}(x)$, and $D$ is a maximal with this property, that is, if for all $x \in D^{\prime} \supset D$ one has $D^{\prime} \subset c l \mathcal{O}^{+}(x)$, then $D^{\prime}=D$. A control set $C \subset M$ is called an invariant control set if $\mathrm{cl} C=\operatorname{cl} \mathcal{O}^{+}(x)$ for all $x \in C$.

We note that every control set $D$ as above is viable in the sense of J.P.Aubin. The following theorem recalls basic properties of control sets. Here a subset $L \subset M$ is called positively invariant, if $\mathcal{O}^{+}(x) \subset L$ for all $x \in L$.

Theorem 1. The system (2.1) has at most countably many control sets and for every $x$ in a compact positively invariant set there is an invariant control set $C \subset$ $\operatorname{cl} \mathcal{O}^{+}(x)$.

If the system is locally accessible, then also the following assertions hold: Every control set $D$ is connected and satisfies cl int $D=$ cl $D$; furthermore, int $D \subset \mathcal{O}^{+}(x)$ for every $x \in D$ and $D=\operatorname{cl} \mathcal{O}^{+}(x) \cap \mathcal{O}^{-}(x)$ for every $x \in$ int $D$. A control set $C$ is invariant iff it is closed. In this case, int $C=\mathcal{O}^{+}(x)$ for all $x \in$ int $C$ and cl int $C=C$; the inclusions int $C \subset \mathcal{O}^{+}(x) \subset C$ hold for all $x \in C$ and, in particular, $C$ is positively invariant. In every compact positively invariant set there are only finitely many invariant control sets.

Most of these properties do not remain valid if the local accessibility condition is violated: There exist invariant control sets $C$ which are not closed and not positively invariant and there are control sets with nonvoid interior which are not connected. Furthermore, the number of control sets on a compact manifold may be countably infinite.

Along with a control set $D$, we study the set of points which can approximately be steered to $D$. This also defines an order on the control sets.

Definition 3. The domain of attraction of a control set $D$ is defined as

$$
\mathbf{A}(D)=\left\{y \in M \mid \operatorname{cl} \mathcal{O}^{+}(y) \cap D \neq \emptyset\right\}
$$

According to Theorem 1, every compact positively invariant set $K$ has the form

$$
K=\bigcup_{i=1}^{l}\left[\mathbf{A}\left(C_{i}\right) \cap K\right]
$$

where $C_{1}, \ldots, C_{l}$ are the invariant control sets in $K$.

Definition 4. The reachability order ${ }^{\prime} \preceq^{\prime}$ on the control sets of system (2.1) is given by

$$
D \preceq D^{\prime} \text { iff } D \cap \mathbf{A}\left(D^{\prime}\right) \neq \emptyset
$$

In fact, this is an order: 
Lemma 1. The relation' $\preceq^{\prime}$ defines an order on the control sets of system (2.1) and

$$
D \preceq D^{\prime} \text { iff } c l \mathcal{O}^{+}(x) \cap D^{\prime} \neq \emptyset \text { for some } x \in D
$$

Proof. First note that this relation does not depend on $x \in D$. This follows by approximate controllability in $D$ and continuous dependence on the initial value. The equivalence to the condition in terms of the domain of attraction is a simple rephrasing. Furthermore, the refexivity condition $D \preceq D$ holds trivially, and $D \preceq$ $D^{\prime}$ and $D^{\prime} \preceq D$ implies $D=D^{\prime}$ by maximality of control sets, i.e. antisymmetry holds. Finally, transitivity follows again by approximate controllability in control sets and continuous dependence on the initial value.

In a compact positively invariant set $K$ where local accessibility holds the invariant control sets coincide with the control sets which are maximal in the reachability order and also with the closed control sets having nonvoid interior. Without local accessibility it is easy to construct maximal control sets which are not invariant.

\section{Relatively Invariant Control Sets}

Now we generalize the results above for subsets $L$ of the state space which are not positively invariant in order to get further insight into the global controllability structure.

Definition 5. For a subset $L \subset M$ a control set $D \subset L$ is called $L$-maximal, if every control set $D^{\prime} \subset L$ with $D \preceq D^{\prime}$ satisfies $D=D^{\prime}$.

Definition 6. For a subset $L \subset M$ a control set $D \subset L$ is called $L$-invariant, if $x \in D$ and $\varphi(t, x, u) \notin D$ for some $t>0$ and $u \in \mathcal{U}$ implies $\varphi(t, x, u) \notin L$.

A generalization of the global description (2.3) for arbitrary sets $L$ instead of compact positively invariant sets $K$ can be given provided that - roughly speaking - a trajectory which starts in $L$ and leaves $L$ cannot return to $L$, local accessibility within $L$ holds, and a further condition holds which allows to fix'the positive orbit within $L$. We introduce the following conditions for a subset $L$ of the state space $M$ :

The no-return condition:

$$
\begin{aligned}
\text { if } \varphi(t, z, u) & \in L \text { for some } z \in c l L, \\
u & \in \mathcal{U}_{p c}, \text { and } t>0, \\
\text { then } \varphi(\tau, z, u) & \in L \text { for all } 0 \leq \tau \leq t
\end{aligned}
$$

and the L-accessibility condition:

$$
\text { for all } y \in L \text { and all } T>0 \text { one has }
$$

$$
\emptyset \neq \operatorname{int}\left[\mathcal{O}_{\leq T}^{+}(y) \cap L\right]
$$

In the following we discuss the existence and the properties of $L$-maximal control sets in a given subset $L \subset M$. Control sets are closed iff they are invariant. For $L$ invariant control sets one obtains an analogous result, provided that the conditions above hold. 
Proposition 1. Consider a subset $L$ be of the state $M$ satisfying (3.1) and (3.2). Then for a control set $D \subset L$ the following properties are equivalent:

(i) the control set $D$ is L-invariant;

(ii) the control set $D$ is closed in $L$, that is, $(\partial D \backslash D) \cap L=\emptyset$.

Proof. If $D$ is $L$-invariant, consider $x \in D$. By (3.2) int $\left[\mathcal{O}_{\leq t}^{+}(x) \cap L\right] \neq \emptyset$ for $t>0$. Now $L$-invariance of $D$ implies that $\mathcal{O}_{\leq t}^{+}(x) \cap L \subset D$, and hence $i n t D \neq \emptyset$. Next suppose that $D$ is not closed in $L$, i.e. there exists $y \in(\partial D \backslash D) \cap L$. By assumption (3.2) we have int $\left[\mathcal{O}_{\leq T}^{+}(y) \cap L\right] \neq \emptyset$ for some $T>0$. Maximality of control sets implies $\mathcal{O}_{\leq T}^{+}(y) \cap D=\emptyset$, since otherwise $y \in D$. Hence there exists $z \in \operatorname{int}\left[\mathcal{O}_{\leq T}^{+}(y) \cap L\right] \backslash D$. Continuous dependence of the solutions on the initial value yields a contradiction to $L$-invariance of $D$.

Conversely, suppose that int $D \neq \emptyset$ and there are $t_{1}>0, x \in D$, and $u \in \mathcal{U}$ with $\varphi\left(t_{1}, x, u\right) \in L \backslash D$. Let $t_{0}:=\sup \{\tau>0 \mid \varphi(\tau, x, u) \in D\}$. By assumption (3.1) we have $\varphi\left(t_{0}, x, u\right) \in L \cap \partial D$. If $\varphi\left(t_{0}, x, u\right) \in D$ one can steer this point into int $D$. Now continuous dependence on the initial value gives a contradiction to maximality of $t_{0}$.

Remark 1. If $L$ is open local accessibility implies the L-accessibility condition (3.2). Furthermore, the no-return condition (3.1) implies that every control set D with $D \cap L \neq \emptyset$ is contained in $L$.

The next proposition shows the existence of a $L$-invariant control set provided that one can fix'the positive orbit in $L$.

Proposition 2. Let $L$ be a subset of the state space $M$ satisfying the conditions (3.1) and (3.2). Consider $x \in L$ and assume that there exists a compact set $Q \subset L$ such that for all $y \in \operatorname{cl} \mathcal{O}^{+}(x) \cap L$

$$
c l \mathcal{O}^{+}(y) \cap Q \neq \emptyset
$$

Then there exists a L-invariant control set $D \subset \operatorname{cl} \mathcal{O}^{+}(x)$ and every L-invariant control set $D \subset \operatorname{cl} \mathcal{O}^{+}(x)$ satisfies cl $D \cap Q \neq \emptyset$.

Proof. For $y \in \operatorname{cl} \mathcal{O}^{+}(x) \cap L$ let $Q(y):=\operatorname{cl} \mathcal{O}^{+}(y) \cap Q$. Consider the family $\mathcal{F}$ of nonvoid and compact subsets in $L$ given by $\mathcal{F}=\{Q(y) \mid y \in Q(x)\}$. Then $\mathcal{F}$ is ordered via

$$
Q(y) \prec Q(z) \text { iff } z \in \operatorname{cl} \mathcal{O}^{+}(y)
$$

Every linearly ordered set $\left\{Q\left(y_{i}\right) \mid i \in I\right\}$ has an upper bound

$$
Q(y)=\bigcap_{i \in I} Q\left(y_{i}\right) \text { for some } y \in \bigcap_{i \in I} Q\left(y_{i}\right)
$$

since the intersection of decreasing compact subsets of the compact set $Q$ is nonempty. Thus Zorns Lemma implies that the family $\mathcal{F}$ has a maximal element $Q(y)$. Now the set

$$
D:=c l_{L}\left[\mathcal{O}^{+}(y) \cap L\right]
$$

is a $L$-invariant control set: Note first that $y \in Q \subset L$, hence $y \in D$ and by (3.2)

$$
\emptyset \neq \operatorname{int}\left[\mathcal{O}_{\leq t}^{+}(y) \cap L\right] \subset D
$$


Thus int $D \neq \emptyset$. Furthermore, every $z \in D$ is approximately reachable from $y$. Conversely, $y \in \operatorname{cl} \mathcal{O}^{+}(z)$, since otherwise $y \notin Q(z)=\operatorname{cl} \mathcal{O}^{+}(z) \cap Q(x)$; hence this is a proper subset of $Q(y)$ contradicting the maximality of $Q(y)$. Hence approximate controllability in $D$ holds. In order to show that $D$ is a control set, we have to verify that $D$ is maximal with this property: Otherwise there is a control set $D^{\prime} \supset D$ containing a point $z \notin D=c l_{L}\left[\mathcal{O}^{+}(y) \cap L\right]$. Clearly, $z \in c l \mathcal{O}^{+}(y)$. The no-return condition (3.1) yields the contradiction $z \in L$ : Steer the system from $z$ into int $D$. Using continuous dependence on the initial value, one can steer the system from $y \in L$ into a neighborhood of $z$ which can be steered into int $D \subset L$. Thus $z \in c l L$ and applying (3.1) again it follows that $z \in L$ contradicting the choice of $z$. Thus $D$ is a control set contained in $L$. Now consider $\varphi(t, z, u) \in L$ with $t>0, u \in \mathcal{U}$, and $z \in D$. Then $\varphi(t, z, u) \in c l_{L}\left\{z^{\prime} \in L \mid z^{\prime} \in \mathcal{O}^{+}(y) \cap L\right\}=D$. Hence $D$ is $L$-invariant.

Finally, note that for every $y$ in a $L$-invariant control set $D \subset \operatorname{cl} \mathcal{O}^{+}(x) \cap L$ one has $\emptyset \neq c l \mathcal{O}^{+}(y) \cap Q \subset L$. Hence $L$-invariance implies $c l D \cap Q \neq \emptyset$.

The proposition above allows us to clarify the relation between $L$-maximal and $L$-invariant control sets and hence yields a characterization of $L$-maximal control sets via their topological properties.

Corollary 1. Let $L$ be a subset of the state space $M$ satisfying the conditions (3.1) and (3.2), let $x \in L$ and suppose that $Q \subset L$ satisfies condition (3.3). Then for a control set $D \subset \operatorname{cl}^{+}(x) \cap L$ the following statements are equivalent:

(i) the control set $D$ is L-maximal;

(ii) the control set $D$ is L-invariant;

(iii) the control set $D$ is closed in $L$, that is, $(\partial D \backslash D) \cap L=\emptyset$.

Proof. The equivalence between (ii) and (iii) follows immediately from Proposition 1. It is easily seen, that statement (ii) implies (i). Conversely, suppose that (i) holds. If there is $y \in D \subset \operatorname{clO}^{+}(x)$ such that $\varphi(t, y, u) \in L \backslash D \subset L \backslash D$ for some $t>0$ and $u \in \mathcal{U}$, then there exists by Proposition 2 a $L$-invariant control set $D_{1} \subset \operatorname{cl} \mathcal{O}^{+}(y) \subset \operatorname{cl} \mathcal{O}^{+}(x)$. Thus $D \preceq D_{1}$ and $D \neq D_{1}$. This contradicts $L$-maximality of $D$. Hence (i) implies (ii).

Next we show that the number of $L$-invariant control sets is finite.

Proposition 3. Let $L$ be a subset of the state $M$ satisfying (3.1) and (3.2) and assume that there is a compact set $Q \subset L$ such that for all $y \in L$ one has $c l \mathcal{O}^{+}(y) \cap$ $Q \neq \emptyset$. Then there are at least one and at most finitely many L-maximal control sets in L. In particular, $L$ has only finitely many connected components. The $L$-maximal control sets coincide with the L-invariant control sets.

Proof. By Corollary 1 the $L$-invariant and the $L$-maximal control sets coincide. Now assume that there are countably many $L$-invariant control sets $D_{n}, n \in \mathbb{N}$. Applying Proposition 2 to $D_{n}$, we obtain that $\operatorname{cl} D_{n} \cap Q \neq \emptyset$. There are $x_{n} \in$ cl $D_{n} \cap Q$ converging to some $x \in Q \subset L$. By Proposition 2 one finds a $L$ - invariant control set $D$ with $D \subset \operatorname{cl} \mathcal{O}^{+}(x)$. Since by Proposition 1 int $D \neq \emptyset$ and we know cl $D_{n}=\operatorname{cl}$ int $D_{n}$, we obtain a contradiction to $L$-invariance of $D_{n}$ for $n$ large enough. Hence there are at most finitely many $L$-invariant control sets.

The second statement follows from the first one, since by the construction in the proof of Proposition 2 one can find a $L$-invariant control set in every connected component of $L$ (recall that control sets are connected). 
Finally, we arrive at the following description of sets $L$ satisfying (3.1) and (3.2).

Theorem 2. Let $L$ be a subset of the state space $M$ satisfying the no-return condition (3.1) and the L-accessibility condition (3.2). Then the following three assertions are equivalent:

(i) There is a compact set $Q \subset L$ such that for all $y \in L$ one has

$$
\operatorname{cl} \mathcal{O}^{+}(y) \cap Q \neq \emptyset
$$

(ii) For all $y \in L$ there is a $L$-maximal control set $D \subset c l \mathcal{O}^{+}(y)$ and there are only finitely many $L$-maximal control sets $D_{1}, \ldots, D_{l_{1}}$.

(iii) The set $L$ can be written as

$$
L=\bigcup_{i=1}^{l_{1}}\left[\mathbf{A}\left(D_{i}\right) \cap L\right]
$$

where $D_{1}, \ldots, D_{l_{1}}$ are the $L$-maximal control sets.

Proof. By Propositions 2 and 3, condition (i) implies (ii). For the converse, choose for every $L$-maximal control set $D_{i}, i=1, . ., l_{1}<\infty$, a point $x_{i} \in \operatorname{int} D_{i}$, and let $Q=\left\{x_{1}, \ldots, x_{l_{1}}\right\}$. Then $Q$ is a compact subset of $L$ and satisfies the condition in (i). The equivalence between (ii) and (iii) follows using Proposition 2.

\section{Applications}

Here we discuss two applications of the theory of relatively invariant control sets developed above. The first one concerns a back stepping'technique for determination of control sets, the second one concerns points which can be steered into different invariant control sets.

Consider the following coupled system which is a special case of (2.1) with controls $u \in \mathcal{U}_{p c}$ on a product space $N \times M$

$$
\begin{aligned}
& \dot{y}(t)=Y(y(t), u(t)) \\
& \dot{x}(t)=X(x(t), y(t), u(t))
\end{aligned}
$$

where $Y: N \times \mathbb{R}^{m} \rightarrow \mathbf{T} N$ and $X: N \times M \times \mathbb{R}^{m} \rightarrow \mathbf{T} M$ are $C^{\infty}$. Note that the second equation does not influence the first one. We will construct control sets for the coupled system from control sets for the first system.

Theorem 3. Assume that the coupled system (4.1), (4.2) on $N \times M$ satisfies the assumptions on (2.1) and that it is locally accessible. Let $D \subset N$ be a control set of the system (4.1) and suppose that $K \subset N \times M$ is a compact positively invariant set such that $D \subset\{y \in N \mid$ there is $x \in M$ with $(y, x) \in K\}$. Then for $L:=(D \times M) \cap K$ there exists a L-invariant control set $D^{\prime}$ and $\{y \in N \mid$ there is $x \in M$ with $\left.(y, x) \in D^{\prime}\right\}=D$.

Proof. In order to deduce the existence of a $L$-invariant control set from Theorem 2 , we have to verify the no-return condition (3.1) and the $L$-accessibility condition (3.2) and we have to construct a compact set $Q \subset L$ such that for all $(y, x) \in L$ one has $\operatorname{cl}^{+}(y, x) \cap Q \neq \emptyset$. Since $D$ is a control set and local accessibility holds, conditions (3.1) and (3.2) hold. Pick an arbitrary element $y_{0} \in$ int $D$ and define $Q:=\left\{\left(y_{0}, x_{0}\right) \in K \mid x_{0} \in M\right\}$. Then the required properties of $Q$ follow. The second assertion follows from the properties of control sets and $L$-invariance of $D$. 
Next we discuss intersections of domains of attraction for control sets. These are critical regions, from which one can steer the system into different parts of the state space. We will show that they are described by the corresponding relatively invariant control sets and their domains of attraction. Fix a compact positively invariant set $K \subset M$ with $K=\operatorname{cl}$ int $K$. Assume that the number $l$ of invariant control sets in $K$ satisfies $2 \leq l<\infty$ and denote them by $C_{i}, i=1, \ldots, l$. The common region of attraction

$$
\mathbf{A}\left(C_{1}, \ldots C_{l}\right)=\bigcup_{i, j \in\{1, . ., l\}, i \neq j}\left[\mathbf{A}\left(C_{i}\right) \cap \mathbf{A}\left(C_{j}\right)\right]
$$

consists of those points which can asymptotically be steered into different invariant control sets. In the following, we often abbreviate

$$
\mathbf{A}:=\mathbf{A}\left(C_{1}, \ldots C_{l}\right) \cap K
$$

The preceding results will be applied to the set $L=\mathbf{A}$. We assume that $\mathbf{A}$ is nonvoid, that local accessibility holds for all points in $\mathbf{A}$, and we require that the following strong invariance condition is satisfied:

For all $x \in K$ there is $\varepsilon_{x}>0$ such that for all $u \in \mathcal{U}$ with $\varphi(t, x, u) \in$ $\mathbf{A}\left(C_{1}, \ldots, C_{l}\right)$ for all $t \geq 0$, the estimate $d(\varphi(t, x, u), \partial L) \geq \varepsilon_{x}$ holds for all $t \geq 0$.

Hence trajectories in $\mathbf{A}$ remain bounded away from $\partial K$. Furthermore, we require that

$$
C_{i} \subset \operatorname{int}_{K}\left[\mathbf{A}\left(C_{i}\right) \cap K\right] \text { for } i=1, \ldots, l
$$

If every point in $K$ is locally accessible, there are only finitely many invariant control sets in $K$ and they satisfy condition (4.3). Observe that here the invariant control sets $C_{i}$ are allowed to have void interior.

In order to show that the no-return condition (3.1) holds and to construct sets $Q_{i}$ satisfying condition (3.4) we will need some topological properties of $\mathbf{A}$. They are guaranteed by assumption (4.3) which requires that $C_{i} \subset$ int $_{K}\left[\mathbf{A}\left(C_{i}\right) \cap K\right]$ for all invariant control sets in $K$.

It is easy to see that under assumption (4.3) the invariant control sets $C_{i}$ in $K$ have an open domain of attraction $\mathbf{A}\left(C_{i}\right) \cap K$ in $K$. Thus also $\mathbf{A}=\mathbf{A}\left(C_{1}, \ldots, C_{l}\right) \cap K$ is open in $K$ and local accessibility in $\mathbf{A}$ implies the $\mathbf{A}$-accessibility condition (3.2).

Let $\partial \mathbf{A}$ and $\partial^{K} \mathbf{A}$ denote the boundaries of $\mathbf{A}$ in $M$ and $K$, respectively, and define

$$
\begin{aligned}
\partial_{j} \mathbf{A} & =\partial^{K} \mathbf{A} \cap \mathbf{A}\left(C_{j}\right), j=1, \ldots, l \\
\text { and } \partial_{0} \mathbf{A} & =\mathbf{A} \cap \partial K
\end{aligned}
$$

Note that each $y \in \partial^{K} \mathbf{A}$ is in the domain of attraction of exactly one control set $C_{i}, i \in\{1, \ldots, l\}$. Since every point in $K$ can approximately be steered into an invariant control set, it follows that

$$
\partial^{K} \mathbf{A}=\bigcup_{i=1}^{l} \partial_{j} \mathbf{A} \text { and } \partial \mathbf{A}=\bigcup_{i=0}^{l} \partial_{j} \mathbf{A}
$$

Using that the domains of attraction are open, one sees that the first union is disjoint; furthermore, $\partial_{j} \mathbf{A}$ is open and isolated in $\partial^{K} \mathbf{A}$ and in the topology of $M$

$$
\begin{aligned}
& y \in c l \partial_{i} \mathbf{A} \cap c l \partial_{j} \mathbf{A} \text { for some } \\
& 1 \leq i, j \leq l, i \neq j, \text { implies } y \in \partial K .
\end{aligned}
$$


Note further that $\partial^{K} \mathbf{A}$ consists of at least two (different) $\partial_{j} \mathbf{A}$. Analogous definitions can be given for every connected component of $\mathbf{A}$ and all properties stated above remain valid.

The following technical lemma will be needed.

Lemma 2. For every $x \in \mathbf{A}$ there are $J \subset\{1, \ldots, l\}$ and $y \in \mathcal{O}^{+}(x)$ such that $y \in \bigcap_{j \in J} \mathbf{A}\left(C_{j}\right)$ and $J$ is a minimal index set in the following sense:

If $\varphi(t, y, u) \in \mathbf{A}$ for some $t>0, u \in \mathcal{U}$, then $\varphi(t, y, u) \in \bigcap_{j \in J_{1}} \mathbf{A}\left(C_{j}\right)$.

Proof. Since $x \in \mathbf{A}$, there exists $J_{1} \subset\{1, \ldots, l\}$ with $x \in \bigcap_{j \in J_{1}} \mathbf{A}\left(C_{j}\right)$. If there are $t_{1}>0$ and $v_{1} \in \mathcal{U}$ with $y_{1}:=\varphi\left(t_{1}, x, v_{1}\right) \in \mathbf{A} \backslash \bigcap_{j \in J_{1}} \mathbf{A}\left(C_{j}\right)$, then there exists a proper subset $\emptyset \neq J_{2} \subset J_{1}$ with $y_{1} \in \bigcap_{j \in J_{2}} \mathbf{A}\left(C_{j}\right)$. Proceeding recursively, one ends up, after finitely many steps, with a point $y \in \mathcal{O}^{+}(x)$ with a minimal index set $J$.

Note that a minimal index set has at least two elements. Furthermore, the lemma implies that for each $\mathbf{A}$-invariant control set $D$ there is $J \subset\{1, \ldots, l\}$ such that for each $x \in$ int $D$ the index set $J$ is minimal.

Proposition 4. Assume that $K \subset M$ with $K=$ cl int $K$ is a compact positively invariant set for the control system (2.1) satisfying the strong invariance condition and condition (4.3) and that the system is locally accessible from every point in $\mathbf{A}$. Let $x \in \mathbf{A} \subset K$ with $x \in \bigcap_{j \in J} \mathbf{A}\left(C_{j}\right)$, where $J \subset\{1, \ldots l\}$ is some minimal index set for $x$. Then there exists a $\mathbf{A}$-invariant control set $D \subset \operatorname{cl} \mathcal{O}^{+}(x)$ with

$$
\partial D \cap \partial_{j} \mathbf{A} \neq \emptyset \text { for all } j \in J
$$

Proof. The proof will be based on Proposition 2. Note that $\mathbf{A}$ is open in $K$, hence local accessibility implies (3.2). Denote the connected component of $\mathbf{A}$ that contains $x$ by $\mathbf{A}_{x}$, and let

$$
Q_{0}:=\left\{y \in \mathbf{A}_{x} \mid d\left(y, c l \partial_{0} \mathbf{A}_{x}\right) \geq \varepsilon_{x}\right\}
$$

where $\varepsilon_{x}$ is chosen according to strong invariance condition. For $\varepsilon>0$ and $j \in J$ define

$$
\begin{gathered}
N_{j}(\varepsilon):=\left\{y \in \mathbf{A}_{x} \| d\left(y, c l \partial_{j} \mathbf{A}_{x}\right) \leq \varepsilon\right\} \\
Q_{j}(\varepsilon):=\left\{y \in \mathbf{A}_{x} \cap Q_{0} \mid d\left(y, c l \partial_{j} \mathbf{A}_{x}\right)=\varepsilon\right\}
\end{gathered}
$$

We can choose $\varepsilon>0$ small enough such that

$$
d\left(\partial_{j} \mathbf{A}_{x} \cap Q_{0}, \partial_{i} \mathbf{A}_{x} \cap Q_{0}\right) \geq 5 \varepsilon \text { for all } i, j \in J, i \neq j
$$

Hence the sets $Q_{j}(\varepsilon)$ are nonvoid, compact, and pairwise disjoint with distance at least $3 \varepsilon$. Decreasing, if necessary, $\varepsilon$ further, we may assume that $x \in \mathbf{A}_{x} \backslash$ $\bigcup_{j \in J} N_{j}(2 \varepsilon)$. Every trajectory $\{\varphi(t, y, u), t \geq 0\}$ with $y \in c l \mathcal{O}^{+}(x) \cap\left(\mathbf{A}_{x} \backslash N_{j}(2 \varepsilon)\right)$ that approaches $C_{j}$ for $t \rightarrow \infty$ must exit through $\partial_{j} \mathbf{A}_{x} \cap Q_{0}$ and must cross $Q_{j}(\varepsilon)$. For every $y$ in this set there exists a control $u \in \mathcal{U}_{p c}$ with this property. Furthermore we find that

$$
\emptyset \neq Q_{j}(x, \varepsilon):=\operatorname{cl} \mathcal{O}^{+}(x) \cap Q_{j}(\varepsilon) \subset \bigcap_{j \in J} \mathbf{A}\left(C_{j}\right)
$$

for all $j \in J$ and all $\varepsilon>0$ small enough. Hence the assumptions of Proposition 2 are satisfied. Finally, assertion (4.5) follows, because in the construction above the number $\varepsilon$ can be made arbitrarily small and every $\mathbf{A}$-invariant control set $D$ has nonvoid intersection with every $Q_{j}(x, \varepsilon)$. 
This proposition together with Lemma 2 immediately yields that the A-invariant control sets coincide with the maximal control sets in the region $\mathbf{A}$. We collect our results in the following theorem.

Theorem 4. Assume that $K \subset M$ with $K=$ clint $K$ is a compact positively invariant set for the control system (2.1) satisfying the strong invariance condition. Furthermore, let $C_{1}, \ldots, C_{l}$ be the invariant control sets in $K$ with $2 \leq l<\infty$ and suppose that they satisfy condition (4.3). Denote

$$
\mathbf{A}\left(C_{1}, \ldots, C_{l}\right)=\bigcup_{i, j \in\{1, . ., l\}, i \neq j}\left[\mathbf{A}\left(C_{i}\right) \cap \mathbf{A}\left(C_{j}\right)\right]
$$

and assume that the system is locally accessible from every point $x \in \mathbf{A}:=\mathbf{A}\left(C_{1}, \ldots, C_{l}\right) \cap$ K. Then

$$
\mathbf{A}=\bigcup_{j=1}^{l_{1}}\left[\mathbf{A}\left(D_{j}\right) \cap K\right]
$$

where $D_{j}, 1 \leq j \leq l_{1}<\infty$, are the $\mathbf{A}$-invariant control sets. The control sets $D_{j}$ can also be characterized either as those control sets in $\mathbf{A}\left(D_{1}, \ldots D_{l}\right)$ which have nonvoid interior and are closed in $\mathbf{A}$; or, as the $\mathbf{A}$-maximal control sets. Furthermore, they are related to the boundary of $\mathbf{A}$ by Proposition 4.

Proof. The number $l_{1}$ of $\mathbf{A}$-invariant control sets satisfies $1 \leq l_{1}<\infty$. Every point in an $\mathbf{A}$-invariant control set $D_{i}$ and hence every point in $\mathbf{A}\left(D_{i}\right) \cap K$ lies in $\mathbf{A}$. Conversely, for every point $x$ in $\mathbf{A}$ there is an $\mathbf{A}$-invariant control set $D_{i}$ with $x \in$ $\mathbf{A}\left(D_{i}\right) \cap K$.

This theorem reduces the computation of the regions $\mathbf{A}=\mathbf{A}\left(C_{1}, \ldots, C_{l}\right) \cap K$ to the computation of finitely many $\mathbf{A}$-invariant control sets and their domains of attraction. Numerical methods for the computation of control sets and their domains of attraction have been developed in [4].

Remark 2. Common domains of attraction are also of interest for random dynamical systems, since they coincide with the regions of multistability, see [1] and $[2]$.

\section{REFERENCES}

[1] F. Colonius, F. J. De la Rubia, and W. Kliemann, Stochastic models with multistability and extinction levels, SIAM J. Appl. Math., 56 (1996), pp. 919-945.

[2] F. Colonius, G. Häckl, And W. Kliemann, Dynamic reliability of nonlinear systems under random excitation, ASME DE, 84-1 (1995), pp. 1007-1024.

[3] F. Colonius and W. Kliemann, Limit behavior and genericity for nonlinear control systems, J. Diff. Equations, 109 (1994), pp. 8-41.

[4] G. HÄCKL, Reachable Sets, Control Sets and Their Computation. Dissertation, Universität Augsburg, Augsburger Mathematische Schriften Band 7, 1996. 